\title{
RESPUESTA DE LA COMUNIDAD DE ARAÑAS EPÍGEAS (ARANEAE) EN LAS LOMAS DE LACHAY, PERÚ, ANTE LA OCURRENCIA DEL EVENTO EL NIÑO 1997-98
}

\section{RESPONSE OF A EPIGEIC SPIDER ASSEMBLAGE (ARANEAE) IN THE NATURE RESERVE "LOMAS OF LACHAY" (LIMA, PERÚ) IN THE FACE OF THE 1997-1998 EL NIÑO}

Alfredo Giraldo ${ }^{1}$, Dante Pérez ${ }^{1}$ y Germán Arellano ${ }^{1}$

\begin{abstract}
Resumen
El presente estudio describe la respuesta de una comunidad de arañas epígeas ante los cambios ambientales provocados por El Niño (EN) 1997-98 en formaciones de lomas de la Costa Central del Perú. La araneofauna fue muestreada durante los años 1998 y 1999 (cuatro veces por año), usando trampas pitfall distribuidas en un área mixta de loma de herbáceas y loma tipo parque en la Reserva Nacional de Lachay. La respuesta de la comunidad fue evaluada analizando los patrones de variación temporal correspondientes a: la composición y abundancia de familias, las abundancias relativas de los gremios, las variables comunitarias (abundancia, biovolumen por individuo, riqueza y diversidad) y las abundancias de las 10 familias más frecuentes. La familia más abundante fue Linyphiidae, incluyendo el $68 \%$ de la abundancia registrada durante los dos años y alcanzando la posición de dominante en seis de las evaluaciones $(>80 \%$ de la abundancia por evaluación). Durante la mayor parte del tiempo de estudio, las tejedoras fueron más abundantes que las vagabundas según los valores de la proporción de abundancia tejedoras:vagabundas. El gremio de las tejedoras estuvo representado mayoritariamente por las tejedoras de suelo (= Linyphiidae) durante ambos años. Los gremios particulares de arañas vagabundas, mostraron diferentes tendencias de variación: las cursoriales en vegetación y las emboscadoras fueron más abundantes durante 1998, mientras que las cursoriales en suelo y las perseguidoras lo fueron durante 1999. Las variables comunitarias fueron notoriamente influenciadas por la dominancia de Linyphiidae durante 1998, a consecuencia de ésta la abundancia se incrementó entre Febrero y Agosto, la diversidad disminuyó en el mismo periodo y asimismo, la riqueza y el biovolumen por individuo disminuyeron en Agosto. Entre las diez familias más comunes hubo diferentes tipos de respuesta: cuatro favorables, tres desfavorables, una indeterminada y dos de relativa indiferencia.
\end{abstract}

Palabras clave: Comunidad de arañas epígeas, El Niño 1997-98, patrones de variación temporal, formaciones vegetales de lomas, Reserva Nacional de Lachay, pitfall.

Abstract
The present study describes the response of epigeic spider assemblages facing environmental changes caused by the 1997-1998 El Niño event in "lomas" formations of the Peruvian central coast. Spiders were sampled during 1998 and 1999 (four times per year), using pitfall traps that were distributed in a mixed area of herbaceous lomas and park type lomas in the Lachay National Reserve. The response of the assemblage was evaluated analyzing temporary variation patterns corresponding to the following: composition and abundance of families, relative abundance of the guilds, community variables (abundance, individual biovolume, richness and diversity) and abundance of the ten most common families. The most abundant family was Linyphiidae, including $68 \%$ of the registered abundance during the two years and reaching a dominant position in six evaluations ( $>80 \%$ of the abundance per evaluation). During most part of the study, web builders were more abundant than the wanderers as is indicated by the values of the web builders:wanderers ratio of abundance. The guild of the web builders was represented mostly by the ground weavers (i.e. Linyphiidae) during both years. Particular guilds of wanderer spiders, showed different variation trends: foliage runners and ambushers were more abundant during 1998, whilst ground runners and stalkers were so during 1999. Community variables were notoriously influenced by the Linyphiidae dominance during 1998. As a consequence, abundance was increased from February to August, diversity decreased in the same period and also, richness and individual biovolume decreased in August. Among the ten most common families there were different kinds of responses: four were favorable, three were unfavorable, one was uncertain and two were of relative indifference.

Key words: epigeic spider assemblage, El Niño 1997-98, temporary variation patterns, lomas vegetal formations, Lachay National Reserve. 


\section{Introducción}

El evento El Niño (EN) consiste en la alteración periódica y cíclica de los patrones habituales de variación de las temperaturas superficiales y nivel del mar en el Océano Pacífico, durante un periodo de 3 años (Arntz \& Fahrbach, 1996). En la costa peruana sus dos manifestaciones físicas más evidentes son: (1) Desplazamiento anormal hasta $\operatorname{los} 10^{\circ}$ a $14^{\circ} \mathrm{S}$ de aguas superficiales tropicales de $30 \mathrm{~m}$ de espesor y (2) Grandes precipitaciones propias de la zona de convergencia intertropical hasta aproximadamente los $7^{\circ} \mathrm{S}$ (Aguilar, 1990).

El término lomas es usado para nombrar a la vegetación dependiente de las neblinas que crece en la región costera del Perú y el Norte de Chile, desde Illescas ( $6^{\circ} 52^{\prime}$ L.S.) hasta La Serena (29 54' L.S.) (Brack, 1976; Dillon \& Rundel, 1990; Dillon, 1997). Dicha vegetación se desarrolla en las laderas orientadas hacia el mar, las cuales favorecen la condensación de las neblinas traídas por los vientos que soplan de Sur y Sur-Oeste, hasta una altitud de 1 $000 \mathrm{~m}$ (Brack, 1976). Durante los últimos dieciocho años, un creciente número de investigaciones ha contribuido al esclarecimiento de las repercusiones que tiene el EN sobre las diferentes comunidades bióticas que habitan en las Lomas. Son destacables al respecto los estudios realizados sobre: flora y vegetación (Dillon \& Rundel, 1990; Cano et al., 1999; Teixeira, 2000; Tovar, 2003), coleópteros (Giraldo, 2002), aves (Velarde, 1983; Tori, 2000; Véliz, 2002) y el zorro andino Dusicyon culpaeus (Falero \& Sánchez, 1988).

El orden Araneae es uno de los grupos más comunes y ubicuos de animales, con la capacidad de prosperar en los más variados hábitats, aun a grandes altitudes (Mani, 1968; Turnbull, 1973). Sus niveles de especiación y abundancia son muy altos, especialmente en los bosques tropicales, con cifras que oscilan ente las 300 y 800 especies por hectárea (Coddington et al., 1991) y hasta un $85 \%$ de la abundancia de los artrópodos inventariados (Basset, 1995). Por otra parte, actúan como los principales reguladores de las poblaciones de insectos (Reichert, 1974) e incluso pueden ejercer una influencia indirecta sobre la tasa de descomposición de materia orgánica en el suelo (Wise et al., 1999; Lawrence \& Wise, 2000). Estos rasgos hacen de las arañas un grupo con gran potencial como indicador de biodiversidad, así como para la caracterización de hábitats y de las disturbancias que actúan sobre ellos (New, 1999).

La información disponible sobre las arañas que habitan en las formaciones de lomas se debe en gran medida a las investigaciones del Dr. Pedro Aguilar, las cuales se concentraron en las lomas de la Costa Central e incluyeron tanto aspectos ecológicos como inventarios de ellas. Entre ellas encontramos evaluaciones generales de Arthropoda (Aguilar, 1954, 1963, 1964, 1976, 1977, 1981, 1985; Aguilar \&
Turkowsky, 1977) evaluaciones de Araneae (Aguilar et al., 1986) y el estudio de la biología de Sicarius peruensis Keys (Aguilar \& Mendez, 1971). Con respecto a las lomas de la Costa Central, cabe añadir la descripción de dos nuevas especies de Salticidae provenientes de los tillandsiales de Lima (Galiano, 1977) y el listado de especies obtenido en la evaluación rápida efectuada por Córdova \& Santisteban (1999) en la Reserva Nacional de Paracas. Para las lomas de la Costa Sur se dispone de un registro de la fluctuación altitudinal de la abundancia de arañas en las Lomas de Mollendo-Matarani (López et al., 1978).

El presente estudio se propone hacer una descripción de los patrones de variación temporal que caracterizaron a la comunidad de arañas epígeas en las lomas de Lachay, durante los años 1998 y 1999, los cuales fueron meteorológicamente influenciados por la ocurrencia de El Niño 1997-98.

\section{Materiales y métodos}

Área de estudio: Los muestreos fueron realizados en la Reserva Nacional de Lachay ubicada a $105 \mathrm{Kms}$. al norte de la ciudad de Lima $\left(11^{\circ} 21^{\prime} 00^{\prime \prime}-11^{\circ} 21^{\prime} 58^{\prime \prime}\right.$ L.S., $77^{\circ} 22^{\prime} 25^{\prime \prime}-77^{\circ} 22^{\prime} 28^{\prime \prime}$ L.O.). De su superficie total de 5070 ha, el área de estudio abarcó aproximadamente unas 430 ha comprendidas entre el Cerro Redondo, la Quebrada Yerbabuena y el Puquial, con un rango altitudinal de 200-600 msnm Este espacio constituye una muestra representativa de las biocenosis denominadas loma de herbáceas y loma tipo parque (Brack, 1976). Durante los dos años de estudio la cobertura vegetal estuvo representada mayoritariamente por las solanáceas Nicotiana paniculata y Urocarpidium peruvianum, condición atribuida al incremento de precipitación provocado por El Niño durante 1998 (Cano et al., 1999; Teixeira, 2000; Tovar, 2003).

La estacionalidad típica de la zona incluye cuatro épocas a lo largo del año (Torres \& López Ocaña, 1981): seca (Enero - Marzo), inicios de época húmeda (Abril - Junio), época húmeda (Julio - Septiembre) e intermedia (Octubre - Diciembre). Durante los años de estudio, los registros de la estación meteorológica de la Reserva indicaron un aumento inusual de la precipitación, mientras que la humedad y la temperatura no superaron el rango de años normales. Los datos de precipitación total mensual de 1998, 1999 y 2000 (este último como referencia de un año normal) pueden observarse en el Anexo 1.

Muestreo: Se realizaron cuatro salidas por cada año en los meses de Febrero, Mayo, Agosto y Diciembre como representativas de las cuatro épocas señaladas anteriormente. Se emplearon trampas pitfall para evaluar la comunidad de arañas epígeas presente en 31 estaciones de muestreo que fueron distribuidas sistemáticamente dentro del área de estudio. Las trampas fueron envases de plástico transparente con 
un diámetro de $9 \mathrm{cms}$ y una capacidad aproximada de $572.5 \mathrm{~cm}^{3}$, enterrados al ras del suelo y llenos a la mitad de su capacidad con una mezcla de 7 partes de agua por 1 de formol al $40 \%$, además de $1 / 4$ de cucharita de detergente. El tamaño de la unidad muestral fue de dos trampas por estación con un esfuerzo temporal de siete días, el mismo que resultó satisfactorio para medir la diversidad de la entomofauna capturada simultáneamente (Ramírez et al., 2002).

Los especímenes adultos fueron determinados a nivel de familia siguiendo las claves de Gerchsman \& Schlapelli (1963) y Nentwig (1993). Aunque la diferenciación de los especimenes en morfoespecies fue necesaria para calcular el biovolumen, se optó por no emplear esta aproximación en el resto del análisis. El uso de morfoespecies en arañas ha demostrado su eficacia en algunos casos, alcanzado una frecuencia de concordancia superior al $80 \%$ con las especies taxonómicas (Oliver \& Beattie, 1993, 1996), sin embargo en otros ensayos apenas ha alcanzado un $50 \%$ de concordancia (Derraik et al., 2002). Estos ensayos coinciden en que este taxón es afectado por la sobreestimación de especies, dado el alto grado de variablidad intraespecífica que lo caracteriza. Con los datos de familias, se corre el riesgo de enmascarar los patrones específicos, sin embargo existe al menos una experiencia anterior en la que los patrones de variación espacial y temporal de la riqueza de familias concordaron con aquellos observados para la riqueza específica (Churchill \& Arthur, 1999). El trabajo fue realizado en el Laboratorio de Control Biológico y Ecología de Artrópodos del Departamento de Biología de la UNALM en donde fueron depositados los especimenes.

Las variables ambientales consideradas fueron de dos tipos: meteorológicas y de vegetación. La precipitación total mensual, temperatura promedio mensual y humedad promedio mensual provienen de los registros de la estación meteorológica de la Reserva. Los datos de densidad de herbáceas (individuos $/ \mathrm{m}^{2}$ ), diversidad gamma de herbáceas (en toda el área de estudio) y diversidad alfa promedio de herbáceas (por estación de muestreo), se obtuvieron mediante evaluaciones realizadas simultáneamente por el Laboratorio de Ecología de Procesos (UNALM). Dichos datos y un análisis detallado de los mismos pueden consultarse en las Tesis de Teixeira (2000) y Tovar (2003).

Análisis de los datos: Para establecer la afinidad entre la composición y abundancias relativas de las familias muestreadas en cada fecha de evaluación, se realizó un análisis cluster con los datos de abundancia de las familias transformados a la escala logarítmica, usando la técnica de unión promedio de clusters (average linkage clustering) y el coeficiente de correlación de Pearson (Krebs, 1989). El coeficiente de Pearson fue escogido, a pesar de sus limitaciones (Krebs, 1989) por su escala de fácil interpretación y porque en el presente caso, brindó un dendrograma equivalente al obtenido con la distancia euclidiana como medida de similaridad.

Las familias de arañas se agruparon en gremios a dos niveles: gremios amplios, tejedoras y vagabundas $\mathrm{y}$, gremios particulares que resultan de la subdivisión de los gremios amplios. Para comparar la abundancia $\mathrm{y}$ el número de familias correspondiente a los gremios amplios se calculó la proporción tejedoras: vagabundas $(\mathrm{T}: \mathrm{V})$. Una vez calculados, los valores fueron transformados a la escala logarítmica, de modo que valores positivos indicarán un predominio de tejedoras, mientras que el caso contrario sea señalado por los valores negativos. El gremio de tejedoras está compuesto por tejedoras de suelo, tejedoras simétricas, tejedoras asimétricas y tejedoras laminares, en tanto las vagabundas incluyen cursoriales en follaje, cursoriales en suelo, emboscadoras y perseguidoras. La denominación de los gremios particulares se basa en las empleadas por Uetz et al. (1999) y Whitmore et al. (2002). La inclusión de cada familia en un gremio se hizo consultando las clasificaciones propuestas por Uetz et al. (1999), Ysnel \& Canard (2000), Toti et al. (2000) y Whitmore et al. (2002). En dichas clasificaciones no se incluye a las familias Ctenizidae, Diguetidae, Loxoscelidae y Oecobiidae, por lo cual su asignación se hizo en base a revisión bibliográfica adicional (Aguilar \& Mendez, 1971; Gerchsman \& Schlapelli, 1963; Nentwig, 1993; Boulton \& Polis, 1999).

La abundancia se presenta a través de sus valores total y promedio por fecha de evaluación. Para este último se calculó un intervalo de confianza usando la distribución t (Steel \& Torrie, 1988). El término abundancia debe ser interpretado como abundanciaactividad, puesto que las capturas efectuadas con trampas de caída (pitfall) reflejan la abundancia y la actividad de los artrópodos capturados en combinaciones desconocidas y variables (Greenslade, 1964; Ausden, 1996).

El biovolumen por individuo se presenta a través de sus valores total y promedio por fecha de evaluación. El biovolumen es una medida del tamaño corporal, que fue empleada por Siemann et al. (1999) para describir las relaciones entre abundancia, diversidad y tamaño corporal existentes en una comunidad de artrópodos de pastizales. Dicha medida es el resultado de multiplicar los promedios de longitud, ancho y grosor de cinco especímenes de cada morfoespecie (a menos que se hayan capturado menos), los cuales fueron medidos usando un vernier. La longitud fue la distancia desde el borde anterior del quelícero a la punta del abdomen. El ancho y el grosor fueron medidos en las partes más anchas o más gruesas respectivamente, sean del prosoma o del opistosoma. Los apéndices no fueron incluidos en las mediciones. Para obtener el valor de biovolumen por 
individuo se dividió su valor entre la abundancia por muestreo o por unidad muestral, según el caso.

La riqueza es el número de familias halladas por fecha de evaluación en toda el área de estudio (total) y en cada unidad muestral (promedio).

La diversidad se calculó empleando el índice de Shannon (Magurran, 1988; Krebs, 1989). El cálculo del índice de Shannon a partir de niveles taxonómicos supraespecíficos se justifica, puesto que la comunidad examinada cumple los requisitos establecidos para ello por Giraldo \& Arellano (2002) es decir: pertenence a un grupo taxonómico amplio (alta probabilidad de capturar varias familias), buena parte de las familias (39\%) incluyen a una sola morfoespecie y su diversidad está determinada por la equidad. Dos niveles espaciales son considerados en el cálculo de la diversidad, toda el área de estudio (diversidad gamma) y cada estación de muestreo (diversidad alfa). Los valores de diversidad alfa sirven para calcular un valor promedio por fecha de evaluación que cuenta con su respectivo intervalo de confianza calculado con la distribución $\mathrm{t}$

Las diez familias más comunes, las cuales incluyeron el $96.6 \%$ de la abundancia total, fueron seleccionadas para examinar la variación temporal de sus abundancias, así como para establecer correlaciones entre éstas y las variables ambientales consideradas.

El análisis cluster y las correlaciones que se requirieron fueron realizados con el programa Statistica versión 6.0.

\section{Resultados y discusión}

Composición y abundancia de familias y gremios: Se capturó un total de 4,404 especímenes incluidos en 23 familias (Tabla 1). La familia más abundante fue Linyphiidae con un $68 \%$ de la abundancia total, seguida por Anyphaenidae y Salticidae, las cuales representaron el $11 \%$ y $4 \%$ respectivamente.

Figura $n^{\circ} 1$. Dendrograma de las 8 fechas de evaluación a partir de las abundancias de las familias muestreadas Coeficiente de correlación de Pearson

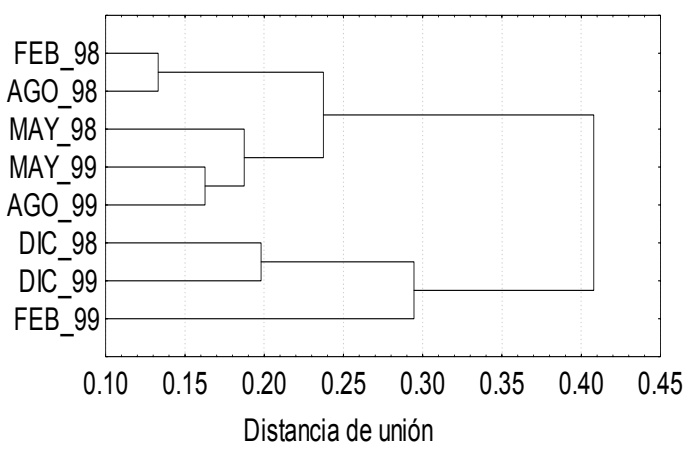

Tabla 1. Abundancias de las familias de arañas colec tadas en la Reserva Nacional de Lachay.Tds= tejedoras de suelo, $\mathrm{T}$ asim= tejedoras asimétricas, $\mathrm{T}$ lam= tejedoras laminares, $\mathrm{C} f=$ cursoriales en follaje, $\mathrm{Cs}=$ cursoriales en suelo, $E=$ emboscadoras, $P=$ perseguidoras.

\begin{tabular}{clccc} 
& & \multicolumn{3}{c}{ Abundancias } \\
Gremios & Familias & 1998 & 1999 & Total \\
Tejedoras & & & & \\
Tds & Linyphiidae & 2010 & 1003 & 3013 \\
T asim & Theridiidae & 70 & 39 & 109 \\
T sim & Araneidae & 13 & 0 & 13 \\
T asim & Loxoscelidae & 1 & 6 & 7 \\
T lam & Agelenidae & 3 & 0 & 3 \\
T lam & Filistatidae & 2 & 0 & 2 \\
T lam & Diguetidae & 1 & 0 & 1 \\
& & 2100 & 1048 & 3148 \\
Vagabundas & & & \\
Cf & Anyphaenidae & 260 & 226 & 486 \\
P & Salticidae & 46 & 118 & 164 \\
Cs & Gnaphosidae & 53 & 77 & 130 \\
Cs & Theraphosidae & 72 & 45 & 117 \\
E & Thomisidae & 45 & 23 & 68 \\
Cs & Lycosidae & 19 & 47 & 66 \\
Cs & Ctenidae & 31 & 26 & 57 \\
Cs & Oecobiidae & 8 & 35 & 43 \\
Cf & Clubionidae & 3 & 29 & 32 \\
Cs & Zodariidae & 11 & 20 & 31 \\
Cs & Oonopidae & 16 & 11 & 27 \\
Cs & Ctenizidae & 2 & 20 & 22 \\
P & Oxyopidae & 5 & 1 & 6 \\
P & Mimetidae & 3 & 1 & 4 \\
Cs & Dysderidae & 1 & 1 & 2 \\
Cs & Sicariidae & 1 & 0 & 1 \\
& & 576 & 680 & 1256
\end{tabular}

La segunda tiene un grado de cohesión menor, con Febrero-1999 separado de las evaluaciones de Diciembre. Dichas agrupaciones responden a cambios en la composición y número de las familias que actuaron como dominantes en cada fecha de evaluación, es decir aquellas que en conjunto incluyeron alrededor del $80 \%$ de la abundancia registrada. En la primera agrupación todas las fechas se caracterizaron porque Linyphiidae fue la familia más abundante, siendo dominante exclusiva de todas las fechas, excepto Mayo-1998 y Agosto-1999, en las cuales compartió su dominancia con Theraphosidae y Anyphaenidae respectivamente. Por lo tanto, en la primera agrupación se observaron como máximo dos familias actuando como dominantes. En la segunda agrupación, Febrero-1999 presentó cuatro familias dominantes, de las cuales Anyphaenidae y Gnaphosidae fueron las más abundantes. En las 
evaluaciones de Diciembre, las familias más abundantes fueron Linyphiidae y Anyphaenidae en ambos casos, sin embargo se distinguieron porque Diciembre-1998 tuvo a Linyphiidae como la familia de mayor importancia numérica y hubo en total tres familias dominantes, en cambio durante Diciembre1999 la familia de mayor importancia numérica fue Anyphaenidae $y$ hubo en total seis familias dominantes.

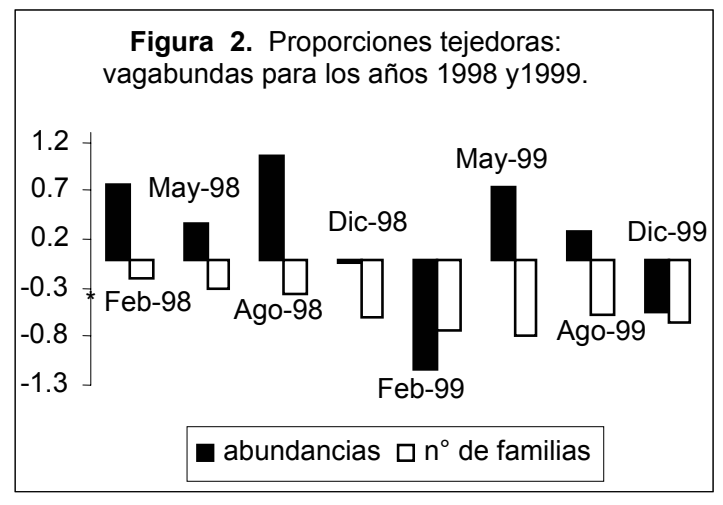

La proporción T:V de abundancias (Figura 2) tuvo su valor máximo en Agosto-1998 y su valor mínimo en Febrero-1999. Predominaron los valores positivos, con lo que se evidencia una superioridad numérica de las tejedoras sobre las vagabundas, excepto para las evaluaciones de Diciembre-1998, Febrero-1999 y Diciembre-1999. En el caso de la proporción T:V del número de familias (Figura 2) el valor máximo correspondió a Febrero-1998 y el valor mínimo a Mayo-1999. Todos los valores fueron negativos, debido al mayor número de familias que componen el gremio de las vagabundas (16) en comparación con el número de familias tejedoras (7). Los mayores valores (más cercanos a cero) correspondieron a las dos primeras evaluaciones, debido a que sólo en ellas fueron registradas las familias de tejedoras simétricas $\mathrm{y}$ tejedoras laminares

El gremio de las tejedoras incluyó seis familias, de las cuales Linyphiidae (tejedoras de suelo) fue la más abundante durante todas las fechas de evaluación, pues incluyó entre el 73 y el $98 \%$ de la abundancia de tejedoras (91\% en promedio). Las familias de tejedoras asimétricas estuvieron presentes ambos años aunque con tendencias opuestas, Theridiidae con mayor abundancia durante el 1998 y Loxoscelidae con mayor abundancia durante 1999. Las cuatro familias integrantes de las tejedoras simétricas y laminares sólo se registraron durante 1998. Con respecto a la familia Araneidae, cabe señalar que su susceptibilidad de captura a través del método de captura empleado es baja, tal y como fuera observado en evaluaciones previas realizadas en lomas de xerófitas (Aguilar \& Turkowsky, 1977; Aguilar, 1977).

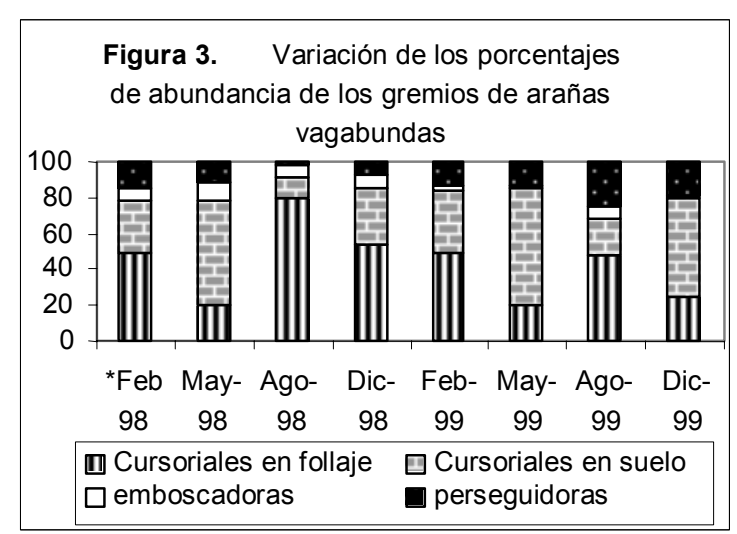

El gremio de las vagabundas incluyó 17 familias, entre las cuales se destacaron Theraphosidae, que incluyó el 39 y $35 \%$ de la abundancia de vagabundas en las evaluaciones de Mayo-1998 y Mayo-1999 respectivamente y Anyphaenidae, que incluyó entre 23 y $76 \%$ de la abundancia de vagabundas en el resto de evaluaciones.

Los gremios particulares de vagabundas presentaron diferencias en los porcentajes de abundancia que representaron a través del tiempo (Figura 3). Las cursoriales en follaje presentaron su menor porcentaje en Mayo-1999 (20\%) y su mayor porcentaje en Agosto-1998 (80\%). Los valores mínimos de ambos años correspondieron al mes de Mayo, los valores máximos correspondieron al mes de Agosto durante 1998 y a los meses de Febrero y Agosto durante 1999 (50 y $48 \%$ respectivamente) asimismo, de un año al otro hubo una disminución en el porcentaje registrado para el mes de Diciembre.

Las cursoriales en suelo presentaron su menor porcentaje en Agosto-1998 (11\%) y su mayor porcentaje en Mayo-1999 (65\%). En ambos años, los valores mínimos corresponden al mes de Agosto y los valores máximos al mes de Mayo, también de un año al otro, hubo un aumento en el porcentaje registrado para los meses de Febrero y Diciembre.

Las emboscadoras presentaron su menor porcentaje en Diciembre-1999 (1\%) y su mayor porcentaje en Mayo-1998 (11\%). Los porcentajes se mantuvieron muy similares durante 1998 y fueron en general mayores a los de 1999, excepto por el registrado en Agosto que fue el de máximo valor para dicho año. 
Tabla $n^{\circ} 2$. Abundancia, biovolumen/ind., riqueza, diversidad y proporciones tejedoras:vagabundas de la comunidad de arañas muestreada durante 1998 y 1999 en las lomas de la Reserva Nacional de Lachay. Intervalos de confianza de las variables comunitarias calculados con la distribución t a un $\alpha=0.05$

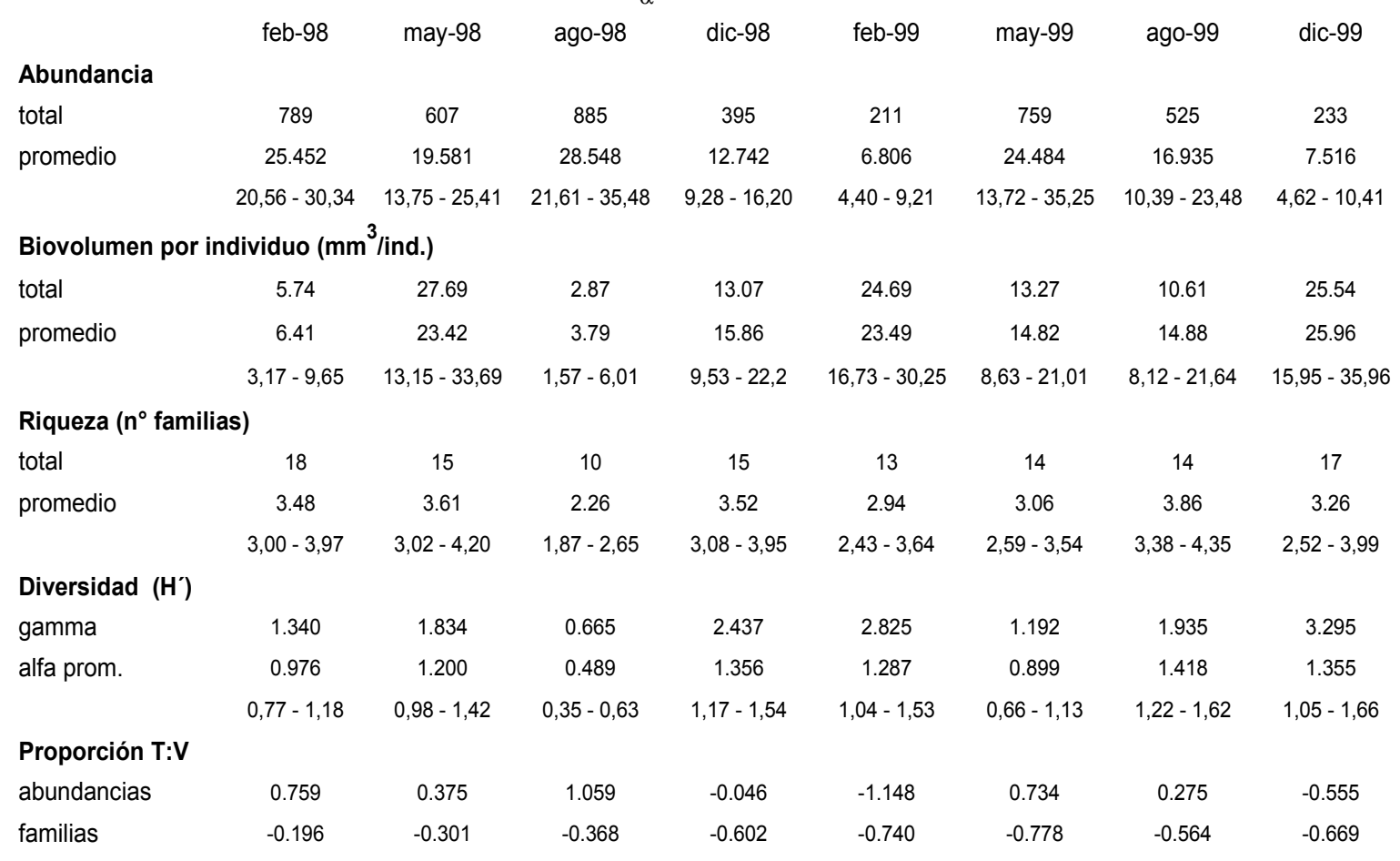

Tabla $\mathbf{n}^{\circ}$ 3. Matriz de correlación para las series temporales de variables comunitarias y proporciones $\mathrm{T}: \mathrm{V}$

$\begin{array}{rccccccc} & \begin{array}{c}\text { Abund. } \\ \text { prom. }\end{array} & \begin{array}{c}\text { Biovol./ind. } \\ \text { prom. }\end{array} & \begin{array}{c}\text { Riqueza } \\ \text { prom. }\end{array} & \text { H'gamma }^{\prime} & \begin{array}{c}\text { H'alfa }^{\prime} \\ \text { prom. }\end{array} & \begin{array}{c}\text { T/V } \\ \text { abund. }\end{array} & \begin{array}{c}\text { T/V } \\ \text { fam. }\end{array} \\ \text { Abund. prom. } & 1 & \mathbf{- 0 . 8 2 3} & -0.276 & \mathbf{- 0 . 9 7 9} & \mathbf{- 0 . 8 1 8} & \mathbf{0 . 9 5 8} & 0.581 \\ \text { Biovol./ind. prom. } & 0.012 & 1 & 0.348 & \mathbf{0 . 8 3 6} & \mathbf{0 . 7 3 8} & \mathbf{- 0 . 7 8 8} & -0.505 \\ \text { Riqueza prom. } & 0.507 & 0.399 & 1 & 0.3526 & \mathbf{0 . 7 7 3 3} & -0.1388 & 0.0952 \\ \text { H'gamma } & 0.000 & 0.01 & 0.392 & 1 & \mathbf{0 . 8 3 6} & \mathbf{- 0 . 9 0 8} & -0.497 \\ \text { H'alfa prom. } & 0.013 & 0.037 & 0.024 & 0.010 & 1 & -0.697 & -0.362 \\ \text { T/V abund. } & 0.000 & 0.02 & 0.743 & 0.002 & 0.055 & 1 & 0.575 \\ \text { T/N fam. } & 0.131 & 0.202 & 0.823 & 0.211 & 0.378 & 0.316 & 1\end{array}$

\footnotetext{
* Los coeficientes de correlación para cada par de comparación se presentan a la derecha de la diagonal, el $p$ valor correspondiente se presenta a la izquierda de la diagonal.
}

* Los coeficientes de correlación en negrita son signficativos a un $\alpha<0,05$

Las cursoriales en suelo presentaron su menor porcentaje en Agosto-1998 (11\%) y su mayor porcentaje en Mayo-1999 (65\%). En ambos años, los valores mínimos corresponden al mes de Agosto y los valores máximos al mes de Mayo, también de un año al otro, hubo un aumento en el porcentaje registrado para los meses de Febrero y Diciembre

Las emboscadoras presentaron su menor porcentaje en Diciembre-1999 (1\%) y su mayor porcentaje en Mayo-1998 (11\%). Los porcentajes se mantuvieron muy similares durante 1998 y fueron en general mayores a los de 1999, excepto por el registrado en Agosto que fue el de máximo valor para dicho año.

Las perseguidoras presentaron su menor porcentaje en Agosto-1998 (1\%) y su mayor porcentaje en Agosto-1999 (24\%). Los porcentajes fueron mayores en 1999 y de un año al otro, los valores aumentaron para los meses de Agosto y Diciembre. Así, cabe señalar que 1998 resultó más favorable para las cursoriales en follaje y las emboscadoras, mientras 
que 1999 lo fue para las cursoriales en suelo y las perseguidoras, siendo destacable la alternancia en el porcentaje que ocuparon los gremios de cursoriales. El muestreo con trampas pitfall pudo haber favorecido la captura de las familias cursoriales, en vista del porcentaje que incluyeron y por el mayor grado de actividad que despliegan al ras del suelo, no obstante cada gremio particular estuvo representado entre las cinco familias más abundantes.

\section{Variación temporal de las variables comunitarias:}

La abundancia promedio (Figura 4) presentó sus valores extremos en cada uno de los dos años, el máximo en Agosto-1998 y el mínimo en Febrero1999. Tomando en cuenta los intervalos de confianza, su variación en el transcurso de los dos años presentó cuatro secuencias de valores: Febrero, Mayo y Agosto de 1998 con valores altos, un descenso durante Diciembre-1998 y Febrero-1999, un ascenso durante Mayo y Agosto de 1999 y, un nuevo descenso durante Diciembre-1999. Su variación intranual fue diferente en cada año, durante 1998 hubo valores altos y sin mayores fluctuaciones en Febrero, Mayo y Agosto seguidos por un descenso en Diciembre, mientras que en 1999 pasó a ser cíclica con un valor bajo en Febrero, un ascenso en Mayo y un retorno a menores valores en Diciembre. Comparando los valores de un año al otro, el único cambio significativo correspondió al mes de Febrero, cuya abundancia promedio descendió de 25.45 a 6.8 individuos capturados por unidad muestral.

Figura $n^{\circ} 4$. Variación temporal de la abundancia promedio durante los años 1998 y 1999

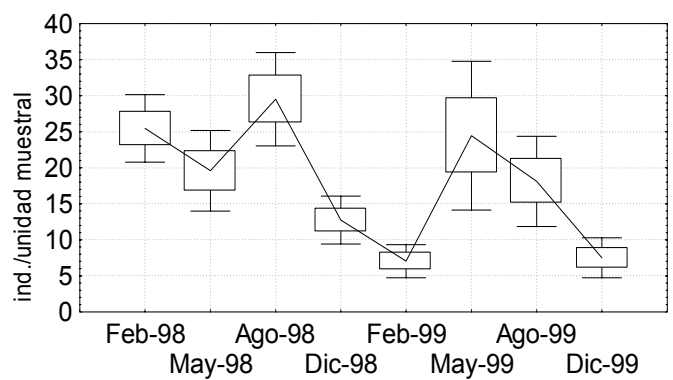

$\mathrm{Su}$ correlación directa y significativa con la proporción $\mathrm{T}: \mathrm{V}$ de abundancias es consecuencia de la dominancia de Linyphiidae, tanto al interior del gremio de las tejedoras, como en la comunidad en su conjunto.

El biovolumen por individuo promedio (Figura 5) presentó sus valores extremos en cada uno de los dos años, el mínimo en Agosto de 1998 y el máximo en Diciembre de 1999. Tomando en cuenta los intervalos de confianza, su variación en el transcurso de los dos años, incluyó cuatro secuencias de valores: Febrero de 1998 con un valor bajo, un ascenso durante Mayo de 1998, un descenso en Agosto de 1998 y un ascenso desde Diciembre de 1998 hasta Diciembre de 1999. $\mathrm{Su}$ variación intranual fue diferente para cada año, con oscilaciones pronunciadas durante 1998 y una mayor constancia durante 1999. Comparando los valores de un año al otro, se observan cambios significativos para los meses de Febrero y de Agosto, cuyos valores de biovolumen promedio por individuo ascendieron de $6.41 \mathrm{~mm}^{3}$ y $3.79 \mathrm{~mm}^{3}$ a $23.49 \mathrm{~mm}^{3}$ y $14.82 \mathrm{~mm}^{3}$ por unidad muestral respectivamente. Esta variable comunitaria presentó correlaciones significativas inversas con la abundancia y con la proporción $\mathrm{T}: \mathrm{V}$ de abundancias. Así, los descensos de Febrero de 1998 y Agosto de 1998 estuvieron provocados por un predominio de arañas de pequeño tamaño (Linyphiidae), mientras que en el resto de muestreos la representación de arañas de mayor tamaño se torno más importante (Por Ej.: Anyphaenidae, Gnaphosidae, Theraphosidae).

Figura $\mathrm{n}^{\circ} 5$. Variacion temporal del biovolumen/ind. promedio durante los años 1998 y 1999

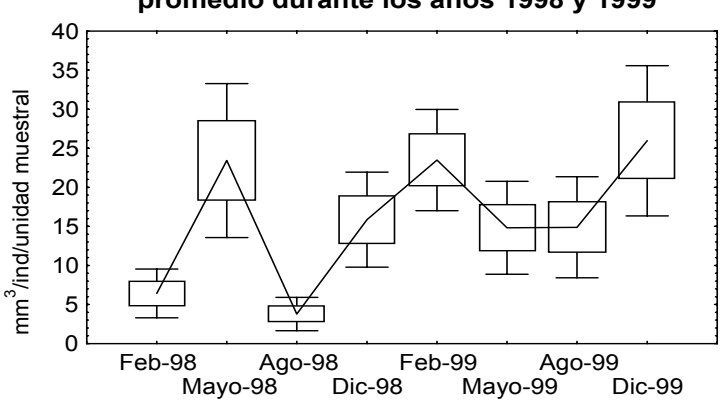

La riqueza promedio presentó sus valores extremos durante los meses de Agosto de cada uno de los dos años, el mínimo en 1998 y el máximo en 1999. Tomando en cuenta los intervalos de confianza, su variación en el transcurso de los dos años, presentó tres secuencias de valores: Febrero y Mayo de 1998 con valores altos, un descenso durante Agosto-1998 y, un ascenso desde Diciembre-1998 hasta Diciembre1999. La variación intranual fue distinta para cada año, durante 1998, se observa un descenso significativo durante el mes de Agosto, mientras que durante 1999 sólo el valor correspondiente al mes de Agosto se insinúa superior aunque no lo es de manera significativa (Figura 6). Comparando los valores de un año al otro, el único cambio significativo correspondió al mes de Agosto, cuya riqueza promedio ascendió de 2.26 a 3.86 familias por unidad muestral.

$\mathrm{La}$ riqueza promedio presentó sus valores extremos durante los meses de Agosto de cada uno de los dos años, el mínimo en 1998 y el máximo en 1999. Tomando en cuenta los intervalos de confianza, su variación en el transcurso de los dos años, presentó tres secuencias de valores: Febrero y Mayo de 1998 con valores altos, un descenso durante Agosto-1998 y, un ascenso desde Diciembre-1998 hasta Diciembre- 
1999. La variación intranual fue distinta para cada año, durante 1998, se observa un descenso significativo durante el mes de Agosto, mientras que durante 1999 sólo el valor correspondiente al mes de Agosto se insinúa superior aunque no lo es de manera significativa (Figura 6). La correlación inversa y significativa con la abundancia promedio, indica que su valor está sujeto en mayor medida a las abundancias relativas de las familias (equidad) y no al número de ellas (riqueza). Como se indicó en la interpretación del dendrograma, la familia que reunió la mayor cantidad de dominancias fue Linyphiidae, así, podría ser que los cambios en la diversidad gamma estuvieron literalmente conducidos por los cambios en la abundancia de dicha familia. Por esta misma razón se correlaciona inversa y significativamente con la proporción $\mathrm{T}: \mathrm{V}$ de abundancias. Su correlación directa y significativa con el biovolumen por individuo promedio refleja la mayor abundancia de arañas grandes durante los muestreos en los cuales se produjeron incrementos en la diversidad.

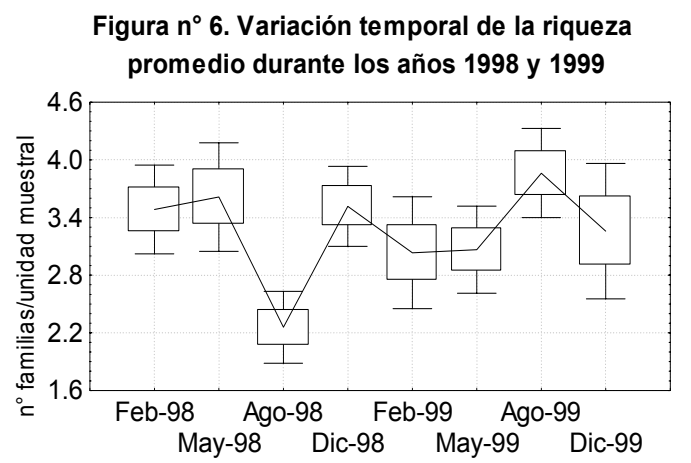

Figura $\mathbf{n}^{\circ}$ 7. Diversidades gamma y alfa promedio para los años 1998 y 1999

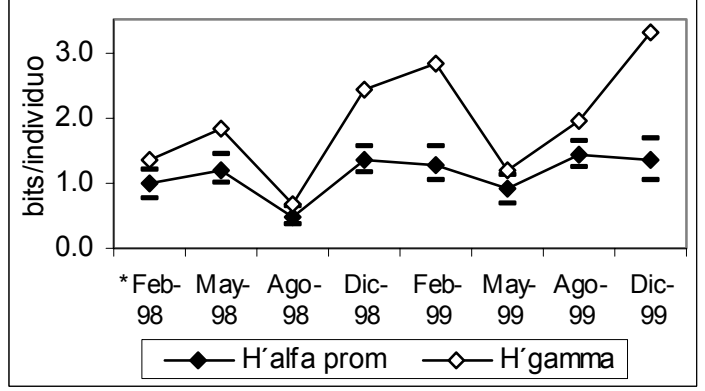

La diversidad alfa promedio presentó sus valores extremos en cada uno de los años, el mínimo en Agosto-1998 y el máximo en Agosto-1999. La variación en el transcurso de los dos años, considerando los intervalos de confianza, presentó cuatro secuencias de valores : Febrero y Mayo de 1998, el descenso de Agosto-1998, un ascenso de Diciembre-1998 a Mayo-1999 y un nuevo ascenso para Agosto y Diciembre de 1999 (Figura 7). Al comparar los valores de un año al otro, se observa que sólo los meses de Agosto no traslaparon sus intervalos de confianza. Esta variable presenta correlaciones significativas con la abundancia promedio (inversa) y con la riqueza promedio (directa), cuyos valores denotan un mayor grado de dependencia con la riqueza en comparación con la equidad. Su correlación directa y significativa con el biovolumen por individuo promedio, queda explicada por el mismo razonamiento expuesto para la diversidad gamma. La correlación directa y significativa con la diversidad gamma queda de manifiesto al comprobar que en la mayoría de fechas de evaluación mostraron las mismas tendencias (ascendentes ó descendentes), en ocasiones con valores muy próximos (Agosto-1998 y Mayo-1999).

La cercanía entre valores de diversidad calculados a escalas espaciales diferentes, indica el tipo de distribución espacial que caracterizó a las especies (o familias) dominantes, uniforme cuando los valores son próximos y agregada cuando los valores se distancian. En el presente caso, los menores valores de diversidad gamma fueron los más próximos a los de diversidad alfa promedio y tal proximidad fue más frecuente al inicio del periodo de estudio (Febrero, Mayo y Agosto de 1998) que hacia el final, a la vez que el distanciamiento entre valores se acentuó (Diciembre1999). Por tanto, la familia que actuó como dominante durante 1998 tendría que haber presentado, no sólo incrementos de abundancia sino también un proceso de expansión dentro del área de estudio. Esto puede verificarse al observar los cambios en el coeficiente de variabilidad espacial de la abundancia de la familia Linyphiidae (Figura 8), el cual aumentó de un año al otro si se realiza una comparación mes a mes y mantuvo una relación directa significativa con la diversidad gamma $(r=0,80 ; P=0.017)$. Se incluyen además los coeficientes de variabilidad espacial de dos familias que también actuaron como dominantes (especialmente cuando Linyphiidae declinó) pero sin embargo no ejercieron una influencia significativa en la tendencia de la diversidad gamma $(\mathrm{r}=0.24 ; \mathrm{P}=$ 0.568 para Anyphaenidae y $\mathrm{r}=-0.45 ; \mathrm{P}=0.265$ para Gnaphosidae) (Figura 8).

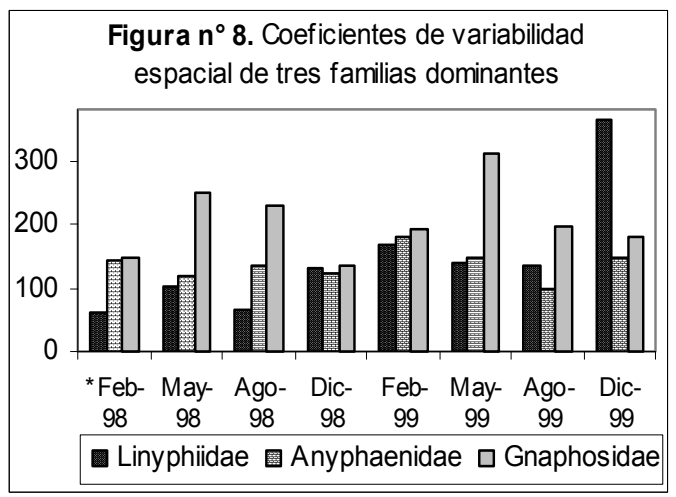


Variación temporal de familias: Entre las diez familias más comunes se encontraron diversos tipos de respuesta frente a la ocurrencia de El Niño:

- Las familias Theridiidae, Ctenidae, Linyphiidae y Thomisidae tuvieron una respuesta favorable con mayores abundancias y/o periodos de actividad anual más largos durante 1998. En 1999, estas familias mostraron la tendencia de concentrar sus abundancias en picos definidos como en Linyphiidae (Mayo) y Thomisidae (Agosto) ó en periodos continuos como en Theridiidae (Mayo a Diciembre). La familia Ctenidae fue la excepción dado que mantuvo una actividad importante durante 1999, con un nivel más bajo en Mayo y los más altos en Agosto y Diciembre (Figuras 9a, b, c y d).

- La familia Anyphaenidae tuvo una respuesta indeterminada puesto que su mayor abundancia se ubicó a la mitad del periodo de estudio (Diciembre1998 y Febrero-1999) siendo superior a la observada tanto para Febrero-1998 como para Diciembre-1999 y asimismo, porque el periodo de actividad anual no fue alterado. Podría tratarse desta favorable tardía o tal vez consecuencia de la combinación de las distintas tendencias que presentaron las morfoespecies integrantes de esta familia (Figura 9e).
- Las familias Salticidae, Lycosidae y Oecobiidae tuvieron una respuesta desfavorable con mayores abundancias y/o periodos de actividad anual más largos durante 1999, siendo destacable el aumento progresivo que muestran sus abundancias desde Febrero-1998 hasta Diciembre-1999. Para Lycosidae y Oecobiidae resulta visible una preferencia por los meses de Febrero y Diciembre, mientras que para Salticidae se presentó una notable actividad durante todo el año con picos en Febrero y Agosto (Figuras 9f, $\mathrm{g}$ y h).

- Las familias Gnaphosidae y Theraphosidae fueron relativamente indiferentes conservando sus patrones de variación anual inalterados, aunque sufriendo cambios de abundancia durante 1998. Para Gnaphosidae las mayores abundancias correspondieron a los meses de Febrero y Diciembre de ambos años, pero con un descenso evidente en Febrero-1998. Para Theraphosidae las mayores abundancias correspondieron al mes de Mayo de ambos años, con un aumento evidente durante 1998 (Figuras 9i y j). Dicho incremento tuvo un efecto notable en el biovolumen por individuo promedio que se registró en dicho muestreo.
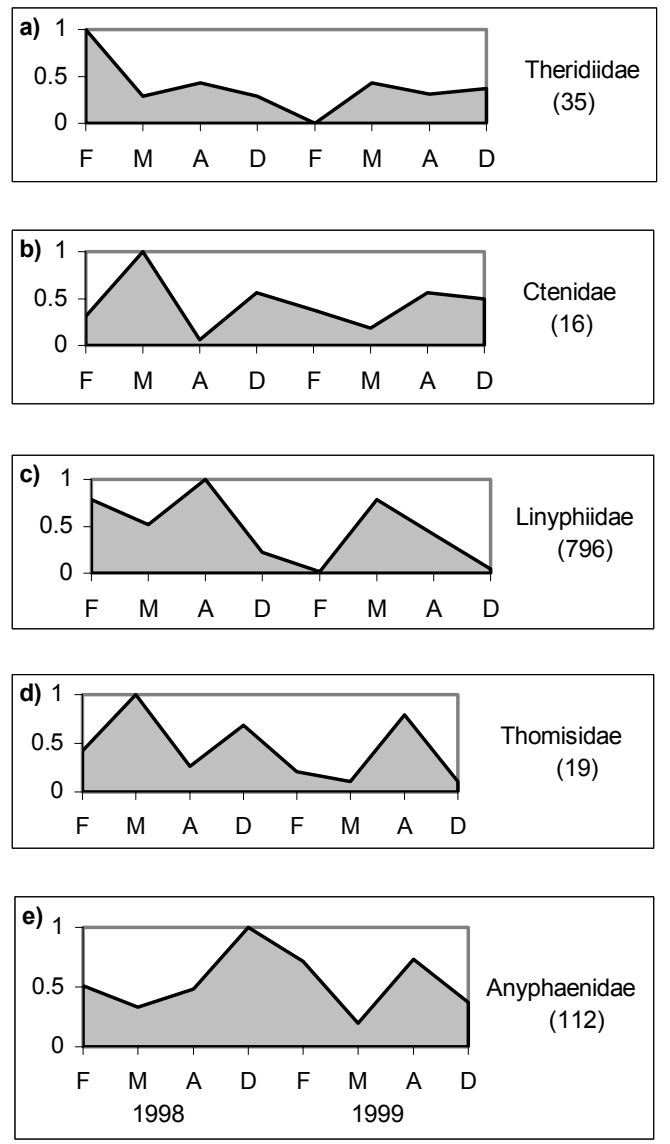
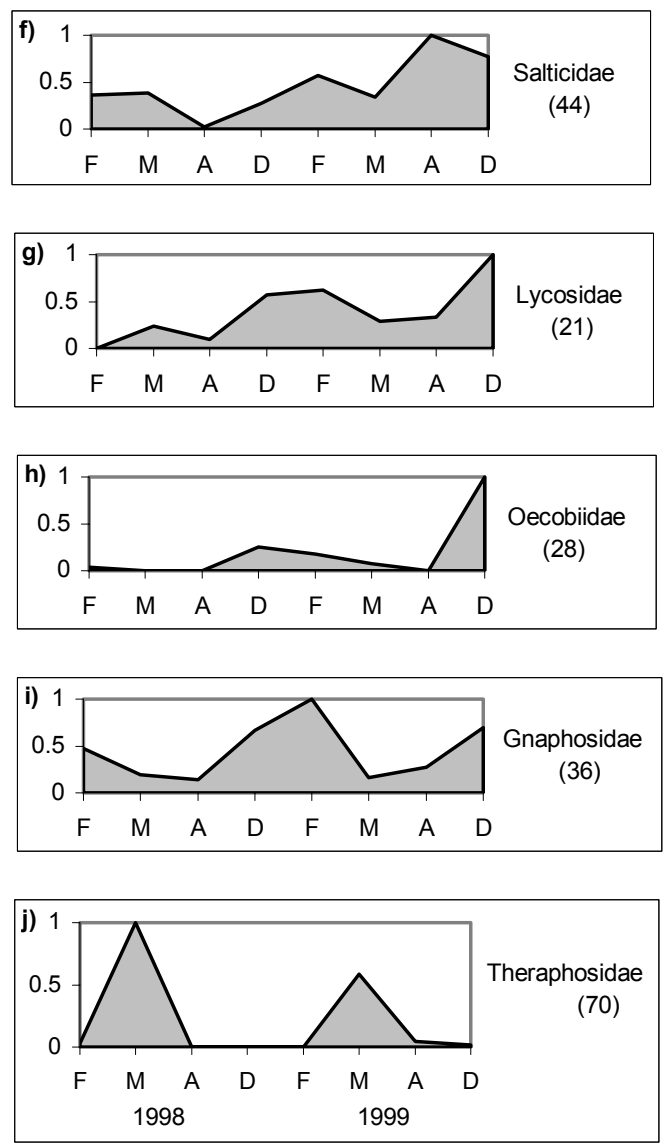

Figura $\mathbf{n}^{\circ}$ 9. Niveles de abundancia como una proporción de la máxima abundancia observada para las familias de arañas más comunes. Números entre paréntesis son la máxima abundancia observada. 
Es importante señalar que los patrones de variación intranual del año 1999 para las familias Ctenidae, Gnaphosidae y Salticidae presentados aquí, son coincidentes ó muy similares a los hallados por Aguilar (1977) en un tillandsial (loma de xerófitas) ubicado a $40 \mathrm{~km}$ al sur de Lima, el cual fue evaluado durante un año de régimen estacional normal. Así, al diferentes estadíos sucesionales, en los cuales la riqueza de plantas y la abundancia de plantas mésicas estuvieron directamente relacionadas con la abundancia de la familia Linyphiidae e inversamente relacionadas con la abundancia de la familia Lycosidae.

Se presentaría una respuesta favorable de las

Tabla $n^{\circ}$ 4. Coeficentes de correlación entre las variables ambientales y la abundancia de las familias de arañas más comunes. Valores resaltados en negrita indican significación a un $\alpha<0,05$

$\begin{array}{rcccccc} & \begin{array}{c}\text { pp. total } \\ \text { mensual }\end{array} & \begin{array}{c}\text { temp. } \\ \text { promedio }\end{array} & \begin{array}{c}\text { humedad } \\ \text { promedio }\end{array} & \begin{array}{c}\text { densidad } \\ \text { herbáceas }\end{array} & \begin{array}{c}\text { div. alfa } \\ \text { herbáceas }\end{array} & \begin{array}{c}\text { div. gamma } \\ \text { herbáceas }\end{array} \\ & (\mathrm{mm}) & \left(\mathrm{C}^{\circ}\right) & (\%) & \left(\mathrm{ind} / \mathrm{m}^{2}\right) & \left(\mathrm{H}^{\prime}\right) & \left(\mathrm{H}^{\prime}\right) \\ \text { Linyphiidae } & 0.696 & -0.343 & 0.507 & 0.642 & \mathbf{0 . 8 0 8} & -0.370 \\ \text { Anyphaenidae } & -0.084 & 0.082 & -0.212 & -0.078 & -0.120 & 0.243 \\ \text { Salticidae } & -0.288 & -0.077 & 0.013 & -0.453 & -0.470 & 0.437 \\ \text { Gnaphosidae } & -0.437 & 0.662 & -0.608 & -0.241 & -0.591 & 0.415 \\ \text { Theraphosidae } & -0.219 & -0.039 & -0.159 & -0.154 & 0.052 & -\mathbf{0 . 7 6 3} \\ \text { Theridiidae } & 0.667 & 0.193 & 0.485 & \mathbf{0 . 8 7 9} & 0.464 & -0.240 \\ \text { Thomisidae } & 0.108 & -0.161 & 0.107 & 0.025 & 0.242 & -0.448 \\ \text { Lycosidae } & -\mathbf{0 . 7 3 5} & 0.123 & -0.289 & -\mathbf{- 0 . 7 2 3} & -\mathbf{0 . 8 9 4} & 0.488 \\ \text { Ctenidae } & -0.316 & 0.101 & -0.139 & -0.308 & -0.251 & -0.357 \\ \text { Oecobiidae } & -0.439 & 0.144 & 0.042 & -0.387 & -\mathbf{0 . 7 2 4} & 0.496\end{array}$

menos para estas familias podría ser que los ciclos de actividad empezaron a retornar a su ritmo habitual al transcurrir un año de la ocurrencia de El Niño 199798.

Para una mejor comprensión de las respuestas de cada familia conviene examinar la relación existente entre la variación temporal de sus abundancias y una serie de variables ambientales (Tabla 4). Se observan correlaciones positivas significativas entre Linyphiidae y la diversidad alfa promedio de herbáceas, así como entre Theridiidae y la densidad de herbáceas. Las correlaciones negativas significativas se establecieron entre: Theraphosidae y la diversidad gamma de herbáceas; Oecobiidae y la diversidad alfa promedio de herbáceas y tambien, Lycosidae con la precipitación total mensual, la densidad de herbáceas y la diversidad alfa promedio de herbáceas. Las familias del gremio de las tejedoras respondieron positivamente al aumento de densidad vegetal y además a la distribución uniforme de la misma dentro del área de estudio (div. alfa prom.), condiciones que proveen del soporte necesario para la construcción de telarañas y favorecen la existencia de microhábitats.

Por el contrario, familias del gremio de las vagabundas con sus correlaciones negativas evidencian preferencia por condiciones en las que proliferan los espacios libres de vegetación, adecuados para sus desplazamientos en busca de presas y con una menor disponibilidad de humedad en el suelo. Relaciones semejantes fueron halladas previamente por Buddle et al. (2000) en bosques de Canadá con familias tejedoras frente a las condiciones ambientales ligadas al aumento de precipitación inducido por El Niño, las cuales son análogas a las de una época húmeda de años normales en tanto, las familias vagabundas podrían presentar una reacción opuesta. Excepciones a estas tendencias generales fueron las observadas por las familias Ctenidae, Theraphosidae y Thomisidae. Sólo en el caso de Thomisidae, podría ser evidente una explicación, estas arañas dependen estrechamente de las flores (y por ende del desarrollo vegetal) para tener acceso a la entomofauna asociada a ellas.

Mención especial merece la familia Linyphiidae dado su rol dominante durante la mayor parte del periodo de estudio. Este grupo de arañas posee una serie de rasgos ideales para actuar como pioneras, los cuales les permiten prosperar en un ecosistema normalmente caracterizado por una marcada estacionalidad y asimismo, aprovechar las condiciones ambientales impuestas por El Niño: En primer lugar, sus cortos ciclos vitales comparados con los de arañas más grandes, les permiten producir varias generaciones al año si las condicionan se tornan favorables para ellas. Al respecto, Draney \& Crossley (1999) comprobaron que las especies de Linyphiidae capturadas en ambientes de menor estadío sucesional tienden a ser multivoltinas y podrían ser capaces de reproducirse oportunísticamente cuando el hábitat se torna favorable o cuando colonizan un hábitat favorable. En segundo lugar, su alta capacidad de dispersión aérea mediante el mecanismo denominado 
aerostación (ballooning), al cual tienen acceso durante todas las fases de su vida dado su pequeño tamaño. Se trata de la familia de arañas que más utiliza este mecanismo de dispersión con probabilidades de éxito mayores a las de otras familias de arañas de mayor tamaño (Suter, 1999). Asimismo, como lo señalan Thomas \& Jepson (1999), este mecanismo está más desarrollado en poblaciones en ambientes impredecibles y les sirve para trasladarse de hábitats de baja calidad a otros de mejor calidad. En tercer lugar, de acuerdo con estudios realizados en campos de cultivo, la dieta de las Linyphiidae está constituida en un $48 \%$ por especies del orden Collembola (Nyffeler, 1999). Dicha preferencia alimenticia podría resultar ventajosa en las condiciones de alta humedad que caracterizan al suelo de las lomas durante la época húmeda y bajo la influencia de El Niño, las mismas que incrementarían la abundancia de Collembola, dado que dicho factor ha sido determinado como influyente en la actividad, densidad y distribución espacial de este taxón (Crawford, 1981; Alvarez et. al., 1999). Sin embargo, la evidencia de una reciente investigación realizada en una formación vegetal dunar de Bélgica, indica que si bien las fenologías de ambos taxa pueden estar correlacionadas, dicha relación estaría subordinada a una preferencia microhábitat común (Bonte \& Mertens, 2003).

Un factor adicional que explicaría la asociación positiva entre Linyphiidae y las condiciones ambientales propiciadas por El Niño, es el pequeño tamaño que caracteriza a sus especies, el cual las haría más susceptibles a la desecación por el incremento de la relación superficie corporal/volumen. Ello contrasta con el mayor tamaño de las arañas cursoriales, las cuales en su mayoría incrementaron sus abundancias a medida que la influencia de El Niño disminuye. Al respecto, Bonte et al., (2000) propusieron este rasgo como una posible explicación para la total ausencia de especies de Erigoninae (Linyphiidae) durante los meses de verano en una formación vegetal dunar de Bélgica. Por otra parte, Ruzicka (2002) al evaluar la araneofauna de unos promontorios rocosos en la Republica Checa, observó que las arañas pequeñas, representadas en su mayoría por Linyphiidae, evitaron las áreas expuestas de tales elevaciones donde el riesgo de desecación es mayor. Dichas áreas fueron mas bien ocupadas por arañas de mayor tamaño, tales como Gnaphosidae y Lycosidae. Si nos acogemos a este planteamiento, el patrón de variación temporal descrito para el biovolumen por individuo adquiere un claro significado biológico.

Sobre los efectos de El Niño 1997-98 en otras poblaciones de arañas, podemos referirnos al seguimiento de dos poblaciones del género Hogna (Lycosidae) efectuado por Baert \& Maelfait (2000) en tres islas del archipiélago de las Galápagos. En años climáticamente normales Hogna albemarlensis habita preferentemente las marismas costeras, estando asociada a los borde de los cuerpos de agua y nunca supera la altitud de $600 \mathrm{~m}$, nivel altitudinal a partir del cual es reemplazada por $H$. galapagoensis, la cual es típica de zonas altas. Como consecuencia de las lluvias frecuentes provocadas por El Niño, las islas se llenaron de charcos permanentes, que permitieron la expansión de $H$. albemarlensis hasta por encima de los $600 \mathrm{~m}$ y en consecuencia, $H$. galapagoensis fue desplazada a altitudes mayores. Salvando las respectivas diferencias en términos de escala espacial y características de hábitat, las dinámicas que fueron observadas en Las Galápagos, guardan alguna relación con las descritas aquí para las arañas epigeas de Lachay.

\section{Conclusiones}

La respuesta de la comunidad de arañas epígeas de las lomas de Lachay ante las precipitaciones provocadas por El Niño 1997-98, tuvo su principal expresión en el incremento de abundancia y consiguiente dominancia de la familia Linyphiidae durante la mayor parte de 1998. Tal dominancia coincidió con un desplazamiento del gremio de vagabundas, las cuales serían el componente mayoritario de la comunidad durante las épocas seca e intermedia, según lo sugiere el patrón observado durante 1999. Sus efectos en las variables comunitarias fueron los incrementos en la abundancia, además de los descensos en el biovolumen por individuo, la riqueza y la diversidad registrados para dicho año.

\section{Agradecimientos}

Al Dr. Edgar Sánchez Infantas, Líder del Proyecto en el marco de la Red de Impacto Biológico "El Niño" RIBEN financiado por CONCYTEC. Al personal de la Reserva Nacional por las facilidades brindadas durante cada muestreo. De manera especial a Beatriz Fuentealba y Karen Eckhardt por su ayuda en el trabajo de laboratorio.

\section{Literatura citada}

Aguilar P. 1954. Estudio sobre las adaptaciones de los artrópodos a la vida en las Lomas de los alrededores de Lima. Tesis Doctoral. Facultad de Ciencias, UNMSM.

. 1963. Los Artrópodos de las Lomas en los alrededores de Lima. Rev. per. Ent. 6(1): 109-114. 1964. Especies de Artrópodos registrados en las Lomas de los alrededores de Lima. Rev. per. Ent. 7(1): 93-95.

1976. Fauna Desértico-Costera Peruana I: Invertebrados más frecuentes en las Lomas. Rev. per. Ent. 19(1): 67-70.

1977. Fauna Desértico-Costera Peruana IV: Artrópodos del Tillandsial de Punta Hermosa, Lima (Perú). Rev. per. Ent. 20(1): 87-92. 
1981. Fauna Desértico-Costera Peruana -

VII: Apreciaciones sobre Diversidad de Invertebrados en la Costa Central. Rev. per. Ent. 24(1): 127-132.

1985. Fauna de las lomas costeras del Perú. Boletín de Lima. 41(7): 17-28.

1990. Sinopsis sobre los eventos del fenómeno "El Niño" en el Perú. Boletín de Lima. 70: 69-84.

\& Mendez M.A. 1971. La "araña chata del nido de arena" Sicarius peruensis (Keys.) 1880: características ecológicas y morfológicas. Rev. per. Ent. 14(1): 143-156.

\& Turkowsky J. 1977. Fauna Desértico-

Costera Peruana - III: Observaciones en el Tillandsial de Cajamarquilla, Lima. Rev. per. Ent. 20(1): 81-85.

, Pacheco V.R. \& Silva T. 1986. Fauna desértico-costera peruana - VIII: arañas de las lomas Zapallal, Lima (nota preliminar). Rev. per. Ent. 29: 99-103.

Alvarez T., Frampton G.K. \& Goulson D. 1999. The effects of drought upon epigeal Collembola from arable soils. Agricultural and Forest Entomology. 1 (4): 243-248.

Arntz W. \& Fahrbach E. 1996. El Niño: Experimento climático de la naturaleza. Fondo de Cultura Económica. México D.F.

Ausden M. 1996. Invertebrates. In: Ecological Census Techniques a handbook. Edited by William Sutherland. Cambridge University Press.

Baert L. \& Maelfait J.P. 2000. The influence of the 1997-1998 El Niño upon the Galápagos lycosid populations, and a possible role in speciation. :5156 In: S. Toft \& Scharff N. eds. European Arachnology 2000. Proceedings of the $19^{\text {th }}$ European Colloquium of Arachnology, Aarhus University Press.

Basset Y. 1995. The taxonomic composition of the arthropod fauna associated with an Australian rainforest tree. Aust. J. Zool. 39: 171-90.

Bonte D. \& Mertens 2003. The temporal and spatial relationship between stenotopic dwarf spiders (Erigoninae: Araneae) and their prey (Isotomidae: Collembola) in coastal grey dunes: A numerical aggregative response or common microhabitat preference? Netherlands Journal of Zoology. 52 (2-4): 243-253.

, Maelfait J.P. \& Hoffmann M. 2000. Seasonal and diurnal migration patterns of the spider (Araneae) fauna of coastal grey dunes. Ekologia (Bratislava). 19(4): 5-16.

Boulton A. \& Polis G. 1999. Phenology and life history of the desert spider, Diguetia mojavea (Araneae, Diguetidae). The Journal of Arachnology. 27: 513-521.

Brack A.J. 1976. Ecología Animal. Primera Parte: Sinecología. Editor Pedro Aguilar F.
Buddle C.M., Spence J.R. \& Langor D.W. 2000. Sucession of boreal forest spider assemblages following wildfire and harvesting. Ecography. 23: 424-436.

Cano A., Roque J., Arakaki M., Arana C., La Torre M., Llerena N. \& Refulio N. 1999. Diversidad florística de las Lomas de Lachay (Lima) durante el evento "El Niño 1997-98". Rev. per. Biol. Vol. Ext.: 125-132.

Churchill T.B. \& Arthur J.M. 1999. Measuring spider richness: effects of different sampling methods and spatial and temporal scales. Journal of Insect Conservation. 3: 287-295.

Coddington J.A., Griswold C.E., Davila D.S., Penagranda E. \& Larcher E.F. 1991. Designing and testing sampling protocols to estimate biodiversity in tropical ecosystems. In: The unity of evolutionary biology (E.C. Dudley, ed.). : 4460. Portland: Dioscorides Press. biology. Oxford: Oxford University Press.

Córdova S. \& Santisteban J. 1999. Evaluación rápida de la biodiversidad de artrópodos en la Reserva Nacional de Paracas, Departamento de Ica, Perú. Informe G.E.A.

Crawford C.S. 1981. Biology of desert invertebrates. Springer-Verlag Berlin Heideberg.

Derraik J.G.B., Closs G.P., Dickinson K.J.M., Sirvid P., Barratt B.I.P. \& Patrick B.H. 2002. Arthropod morphospecies versus taxonomic species: a case study with Araneae, Coleoptera and Lepidoptera. Conservation Biology. 16(4): 1015-1023.

Dillon M. 1997. Lomas Formations-Peru. En: Centres of Plant Diversity, A Guide and Strategy for their Conservation. WWF Information Press, Oxford, U.K.

\& Rundel P. 1990. The botanical response of the Atacama and peruvian desert floras to the 1982-83 El Niño Event. En: Global Ecological Consequences of the 1982-83 El Niño-Southern Oscillation. Glynn, P.W. (ed.) Elsevier, New York.

Draney M. \& Crossley D.A. 1999. Relationship of habitat age to phenology among ground-dwelling linyphiidae (araneae) in the southeastern united states. The Journal of Arachnology. 27: 211-216.

Falero M. \& Sánchez E. 1988. Comportamiento alimentario del zorro andino (Dusicyon culpaeus) en la Reserva Nacional de Lachay. Zonas Aridas. (5): 99-125.

Galiano M.E. 1977. Fauna desértico-costera peruanaII: Dos nuevas especies de Salticidae (Araneae) de los tillandsiales de Lima. Rev. per. Ent. 20(1): 7780 .

Gerchsman De Pikelin B. \& Schlapelli R. 1963. Llave para la determinación de familias de arañas argentinas. Physis. XXIV (67): 43-72.

Giraldo A. 2002. Análisis de los patrones de variación espacio-temporal de las poblaciones de coleópteros en la Reserva Nacional de Lachay durante el 
periodo 1998 - 2001. Tesis para optar el Título de Biólogo - UNALM.

\& Arellano G. 2002. Equivalencia entre series temporales de diversidad para dos niveles taxonómicos. Ecol. Apl. 1(1): 43-49.

Greenslade P.J.M. 1964. Pitfall traping as a method for studying populations of carabidae. Journal of Animal Ecology. 33: 301-310.

Krebs J. 1989. Ecological Methodology. University of British Columbia. Harper Collins Publishers.

Lawrence K.L. \& Wise D.H. 2000. Spider predation on forest-floor Collembola and evidence for indirect effects on decomposition. Pedobiologia. 44: 33-39.

López E., Nuñez J. \& Dávila J. 1978. Fauna Desértico-Costera Peruana VI: Artrópodos de las Lomas de Mollendo-Matarani (Arequipa). Rev. per. Ent. 21(1): 31-38.

Magurran A. 1988. Diversidad Ecológica y su Medición. Ediciones Vedra S.A.

Mani M.S. 1968. Ecology and biogeography of high altitude insects. Dr. Junk N. V. publishers - The Hague.

Nentwig W. 1993. Spiders of Panama. Biogeography, investigation, phenology, checklist, key and bibliography of a tropical spider fauna. Flora \& Fauna Handbook $N^{\circ} 12$. Sandhill Crane Press. Inc. Gainesville, Florida, USA.

New T.R. 1999. Untangling the web: spiders and the challenges of invertebrate conservation. Journal of Insect Conservation. 3: 251-256.

Nyffeler M. 1999. Prey selection of spiders in the field. The Journal of Arachnology. 27: 317-324.

Oliver I. \& Beattie A.J. 1993. A possible method for the rapid assessment of biodiversity. Conservation Biology. 7(3): 562-568.

1996. Invertebrate morphospecies as surrogates for species: A case study. Conservation Biology. 10(1): 99-109.

Ramírez D., Pérez D., Sánchez E. \& Arellano G. 2002. Esfuerzo de muestreo para la evaluación de la diversidad colectada en pitfall en la Reserva Nacional de Lachay-Perú. Ecol. apl. 1(1): 37-42.

Reichert S. 1974. Thoughts on the ecological significance of spíders. BioScience. 24(6): 352356.

Ruzicka V. 2002. Spatial distribution of spiders (Araneae) on scree slopes in Krivoklatsko and Moravsky Kras protected landscape areas. Acta Soc. Zool. Bohem. 66: 321-328.

Siemann E., Tilman D. \& Haarstad J. 1999. Abundance, diversity and body size: patterns from a grassland arthropod community. Journal of Animal Ecology. 57: 713-724.

Steel R. \& Torrie J. 1988. Bioestadística: Principios y procedimientos. Mc Graw-Hill / Interamericana de México S.A. de C.V.
Suter R. 1999. An aerial lottery: the physics of ballooning in a chaotic atmosphere. The Journal of Arachnology. 27: 281-293.

Teixeira V. 2000. El efecto del evento El Niño en la variación de la diversidad de la vegetación herbácea de la Reserva Nacional de Lachay. Tesis para optar el Título de Bióloga-UNALM.

Thomas C.F.G. \& Jepson P.C. 1999. Differential aerial dispersal of linyphiid spiders from a grass and a cereal field The Journal of Arachnology. 27: 294-300.

Tori W. 2000. Análisis de los patrones de variación espacio-temporal de las poblaciones de aves de la Reserva Nacional Lachay. Tesis para optar el Título de Biólogo-UNALM.

Torres J. \& López Ocaña C. 1981. Productividad primaria en las Lomas de la Costa Central del Perú. Separata del Boletín de Lima. (14): 1-11.

Toti D., Coyle F. \& Miller J. 2000. A structured inventory of appalachian grass bald and heath bald spider assemblages and a test of species richness estimator performance. The Journal of Arachnology. 28: 329-345.

Tovar C. 2003. Análisis de la Resiliencia de las comunidades herbáceas de las Lomas de Lachay, provincia de Huaura, Departamento de Lima después de su perturbación por el evento El Niño 1997-1998. Tesis para optar el Título de Bióloga UNALM.

Turnbull A.L. 1973. Ecology of the true spiders (Araneomorphae). Ann. Rev. Entomol. 18: 305348.

Velarde D. 1983. Evaluación de la fauna de vertebrados de las lomas de Iguanil. Zonas Aridas. (3): 83-90.

Véliz C. 2002. Resiliencia de comunidades de aves en la Reserva Nacional de Lachay luego del evento El Niño 1997 - 98. Tesis para optar el Título de Bióloga-UNALM.

Whitmore Ch., Slotow R., Crouch T. \& DippenaarSchoeman A. 2002. Diversity of spiders (Araneae) in a savanna reserve, northern province, south Africa. The Journal of Arachnology. 30:344-356.

Wise D.H., Snyder W.E. \& Tuntibunpakul P. 1999. Spiders in decomposition food webs of agroecosystems: theory and evidence. The Journal of Arachnology. 27: 363-370.

Uetz G., Halaj J. \& Cady A. 1999. Guild structure of spiders in major crops. The Journal of Arachnology. 27: 270-280.

Ysnel F. \& Canard A. 2000. Spider biodiversity in connection with the vegetation structure and the foliage orientation of hedges. The Journal of Arachnology. 28:107-114. 


\footnotetext{
${ }^{1}$ Laboratorio de Control Biológico y Ecología de Artrópodos del Departamento Académico de Biología. Universidad Nacional Agraria La Molina. Av. La Molina s/n Apartado 12-056 Lima 12 Perú. Correo electrónico: Giraldo troodon76@hotmail.com / .Pérez daheps@latinmail.es

/Arellano acg@lamolina.edu.pe
} 